\title{
Der heilige Gerhard, Bischof von Tschanad
}

\author{
I. Zusammenfassung. - II. Biographie. - III. Der liturgische Kult. - IV. Gedenk- und Kultstätten. - V. \\ Auswahlbibliographie.
}

\section{Zusammenfassung}

Der heilige Gerhard ist der erste Märtyrerbischof Ungarns, eine symbolische Gestalt des ungarischen Christentums. Sein mittelalterlicher Kult war weniger bedeutend als der der Heiligen aus dem Árpádenhaus, aber in der Galerie der ungarischen Heiligen war er immer vorhanden. Seine Hauptkultstätten in Ungarn wurden im Laufe der Jahrhunderte zerstört. Erst ab dem 19. Jahrhundert entstanden aufgrund kleinerer Reliquien, die aus Murano zurückerhalten werden konnten, neue Erinnerungsstätten. Das Verhältnis der politischen Macht zu Gerhard ist seit dem Ende des 19. Jahrhunderts abhängig von deren jeweiliger Stellung zum Christentum. Ihn zu feiern - mitunter auch auf recht demonstrative Weise - wurde im 20. Jahrhundert anläßlich der Jubiläen möglich. Seit dem Systemwechsel von 1989 erneuerte sich mit dem auflebenden Kult der ungarischen Heiligen auch die Gerhard entgegengebrachte religiöse Verehrung.

\section{Biographie}

Das Leben Gerhards (ungarisch Gellért, um 980 - 24. September 1046) ist vor allem aus hagiographischen und liturgischen Quellen bekannt. Da die Zahl dieser Quellen klein ist und die Werke überdies nicht eindeutig datierbar sind, gibt es nur wenige belegbare Angaben zu seinem Leben und Wirken. Auf Gerhards italienische Herkunft weisen seine beiden Legenden hin. Dagegen erzählt nur die interpolierte Legenda maior, daß er ein Abkömmling der reichen venezianischen Patrizierfamilie Sagredo war und auf Wunsch seiner Eltern in das Benediktinerkloster San Giorgio gekommen sei, wo man ihn nach seinen Studien zum Prior und später zum Abt gewählt habe. Möglicherweise vertrat Gerhard eine vom heiligen Romuald beeinflußte und mit dem Eremitentum sympathisierende Benediktinerrichtung. Während seiner Pilgerreise nach Jerusalem war Gerhard um 1020, zur Herrschaftszeit Stephans I., nach Ungarn gekommen. Auf Ansuchen des Königs hin unterbrach er seine Reise und wurde der Legenda maior nach zum Erzieher des Thronfolgers Emerich. Andere Quellen, wie die Legenden der heiligen Stephan und Emerich, belegen dies allerdings nicht. Gerhard lebte lange Zeit als Eremit in der Nähe des Klosters Bél im Bakonygebirge nördlich des Plattensees, was beide Legenden bezeugen. Von dort berief ihn der König an die Spitze des 1030 gegründeten Bistums Maroschburg. Die Legenda maior schildert ausführlich, wie Stephans Heeresführer Csanád den sich gegen den König auflehnenden Stammesfürsten Ajtony im Südosten des Landes 
besiegt und dessen Herrschaftsgebiet dem direkten Einfluß des Königs unterstellt hatte. Das Zentrum des Territoriums des vom byzantinischen Kaiser getauften Ajtonys war Maroschburg, das nach Ajtonys Niederlage den Namen Csanáds erhielt. Beide Namensformen wurden lange Zeit für Stadt und Bistum abwechselnd verwendet.

Die Organisation des Bistums Maroschburg/Tschanad, das teilweise noch heidnisch war, bedeutete eine große Herausforderung, der Gerhard allerdings gewachsen war. Er erbaute an der Marosch einen Bischofsdom, den er dem Patrozinium des heiligen Georg unterstellte, und gründete ein Benediktinerkloster zu Ehren der Jungfrau Maria, das er als seine Beerdigungsstätte bestimmte. Gerhards Marienverehrung, die von beiden Legenden betont wird, ähnelt der von König Stephan I., der sein Land dem Schutz der Jungfrau Maria anempfahl. Gerhard hatte mit Sicherheit Einfluß auf den König ausgeübt, und so konnten beide Initiatoren des besonderen Marienkults in Ungarn werden. Gerhard gründete in Maroschburg/Tschanad neben dem Dom eine Schule, organisierte die Priesterausbildung, predigte und taufte unermüdlich. Obwohl er an der Ostgrenze des lateinischsprachigen Europa wirkte, pflegte er nachweislich Kontakte zu dessen Zentren. So widmete er seine theologische Erörterung Deliberatio supra hymnum trium puerorum einem deutschen Benediktiner namens Isingrim. Die Deliberatio kommentiert das im Offizium gesungene Canticum, den Lobgesang der drei Jünglinge im Feuerofen (Dan 3, 57-65). Das Werk ist mehr eine kontemplative, mystische Meditation als ein theologischer Traktat, ganz singulär in Stil, Grammatik und Wortgebrauch. Gerhards in der Deliberatio genannten übrigen Werke sind verloren gegangen.

Als Bischof nahm Gerhard an der Landespolitik teil. In den Thronzwistigkeiten nach dem Tod Stephans I. trieb ihn sein Gerechtigkeitsempfinden in eine offene Konfrontation zum neuen König. Zum Verhängnis wurde ihm der 1046 ausgebrochene Heidenaufstand, der die inneren Machtkämpfe begleitete. Nach der Legende wurde er zusammen mit drei anderen Oberhirten, die sich nach Pest begaben, um die aus der Verbannung zurückgerufenen Herzöge András und Levente zu empfangen, von den Heiden an der Donaufähre auf der Ofener Seite am 24. September 1046 getötet. Gerhards Leichnam überführte man einige Jahre später nach Tschanad und bestattete ihn seinem Willen gemäß im Benediktinerkloster.

1083 wurde Gerhard auf Betreiben von König Ladislaus I. zusammen mit dem Staatsgründer, König Stephan I., dessen Sohn Emerich und den Eremiten András und Benedek von Papst Benedikt IX. heiliggesprochen. 1092 erklärte die Synode von Szabolcs unter dem Vorsitz des Königs den Todestag Gerhards, den 24. September, zum Feiertag.

\section{Der liturgische Kult}

Eine Voraussetzung des liturgischen Kults des Heiligen ist seine Legende in Form von liturgischen Lesungen für die Nokturnen seines Festes, doch auch abgesehen von der Liturgie ist die Legende eine Hauptstütze des Heiligenkults. Für den Kult ist die historische Authentizität der Legende, wie sie von der Nachwelt in der Regel verlangt wird, 
kein erstrangiger Gesichtspunkt. Über den heiligen Gerhard sind zwei miteinander verwandte Legenden erhalten: eine in liturgische Lesungen eingeteilte kürzere Variante, in der Fachliteratur als Legenda minor bekannt (Passio beatissimi Geradi, BHL 3426), und eine romanhafte Legendenredaktion, bestehend aus 28 Kapiteln, die Legenda maior (De sancto Gerhardo episcopo Morosiensi et martyre regni Hungarie, BHL 3424). Um die Datierung der beiden Legenden, ihr Verhältnis zueinander und zu den ungarischen Chroniken des 13. und 14. Jahrhunderts besteht seit vielen Jahrzehnten ein Streit unter Historikern und Philologen. Die Frage ist, ob die knapp gehaltene Legenda minor die glaubwürdigere, frühe Quelle ist, die man später romanhaft erweiterte, oder ob es sich bei der Legenda maior um die ursprüngliche Variante handelt, die für den liturgischen Gebrauch verkürzt und im 14. Jahrhundert modernisiert wurde. Die Glaubwürdigkeit der Legenda maior war zu Beginn des 20. Jahrhunderts noch unumschränkt. Sie diente als Basis für die ersten Monographien über Gerhard; in der breiteren Öffentlichkeit dominiert das darin zum Vorschein kommende Bild des Heiligen bis heute.

Das Gewicht des liturgischen Kults eines Heiligen zeigt sich daran, wie viele eigene Texte sich in den Antiphonen, Responsorien oder Hymnen des Offiziums befinden. Bisher schien es, als sei Gerhards liturgischer Kult in Ungarn nicht bis zu einem eigenen Offizium gediehen, einzig eine magnificat-antiphon war bekannt (A progenie in progenies); die übrigen Texte waren dem commune martyrium entnommen. Allerdings sind die mittelalterlichen liturgischen Quellen des Bistums Tschanad vollständig vernichtet, so daß nichts über die Praxis des liturgischen Kults bekannt ist. Gerhards einziges bisher bekanntes Reimoffizium entstand in Venedig gemäß der „Kleineren Legende“ für den Dom San Marco (Analecta Hymnica 45a). Kürzlich konnte jedoch in einem Brevier, abgefaßt im Zagreber Ritus des 14. Jahrhunderts, ein unbekanntes Gerhard-Offizium entdeckt werden. Wie Tschanad gehörte auch Zagreb zum Erzbistum Kalocsa. Unter den Antiphonen des niveauvollen Reimoffiziums stammen sechs aus der Legenda maior oder deren früheren Varianten und stellen Gerhard als Emerichs Erzieher und einen in den Kirchengesetzen und der Theologie erfahrenen Benediktinermönch dar; sie beschreiben auch Gerhards Tod. Das im Brevier enthaltene Responsorium wiederum weist auf Gerhards Aufenthalt in Gallien hin, was bisher nur in der Deliberatio vorgekommen war. Die Herausgeber des wiederentdeckten Textes, Andrea Kovács und Miklós Földváry, gehen davon aus, daß das Offizium wahrscheinlich dem Tschanad-Kult des heiligen Gerhard im 14. Jahrhundert diente, was von der Königin Elisabeth, Witwe König Karl Roberts und Verehrerin Gerhards, angeregt worden war.

In gedruckten Brevieren des ausgehenden Spätmittelalters finden sich verkürzte Varianten der „Kleineren Legende“, doch war die Legenda minor auch außerhalb des liturgischen Rahmens bekannt. Für die Legenda aurea des Jacobus de Voragine, ein populäres Legendarium des 13. Jahrhunderts, entstand am Ende des 15. Jahrhunderts eine ungarische Ergänzung, die als Legenda sanctorum regni Hungarie in Lombardica historia non contente zweimal gedruckt wurde: in Straßburg um 1486 und in Venedig 1498. In diesen Druckwerken ist Gerhard durch die Legenda minor vertreten. Daß allerdings Gerhard in den Predigtsammlungen der beiden bedeutendsten ungarischen Franziska- 
nerprediger Pelbárt von Temesvár und Osvát von Laskó keinen eigenen Sermon erhielt, zeigt dessen eingeschränkte Popularität. Lediglich in Pelbárts Pomerium de sanctis folgt erst nach der 65. Predigt eine Gerhard-Legende, ein Auszug aus der Legenda maior. $\mathrm{Zu}$ Predigtzwecken war diese farbenfrohe Legende geeigneter als die Legenda minor. Der Band erschien zwischen 1499 und 1521 insgesamt zwanzigmal von Hagenau über Straßburg bis Paris und Lyon. Aus dem Jahr 1511 ist auch eine etwas bearbeitete Krakauer Ausgabe bekannt. 1526 wurde Pelbárts Version schließlich erstmals ins Ungarische übersetzt. Damals stellte ein Kartäuser ein Predigtbuch und Legendarium für Nonnen und Laienbrüder, die in der Regel der lateinischen Sprache nicht mächtig waren, in ungarischer Sprache zusammen. In diesen sogenannten Érdy-Kodex nahm er auch Pelbárts Version der Gerhard-Legende auf.

Fast gleichzeitig mit der ungarischen Übersetzung erfuhr die Legenda maior eine Überarbeitung ganz anderer Art. 1519 erschien das Büchlein Divus Gerardus episcopus et martyr von Giulio Simone, einem in Rom tätig gewesenen sizilianischen Humanisten. Aus dem Widmungsbrief für den kleinen Band geht hervor, daß der Tschanader Domherr Clemens in einem sehr alten Buch („,iber antiquissimus“) auf Gerhards Lebenslauf gestoßen sei. Der Inhalt des Textes gefiehl ihm ungemein, doch empfand er den Stil des lateinischen Textes als barbarisch (,,barbarie et tenebris latinitata“) und wandte sich an Simone, Lektor der Universität Rom; dieser sollte den Stil des Textes verschönern, ganz im Sinn des Renaissance-Geschmacks, der am Tschanader Bischofssitz unter Bischof Miklós Csáki Verbreitung gefunden hatte.

Die einzige erhaltene bildliche Darstellung der Gerhardlegende ist dem Hof der Anjoukönige zu verdanken. Um 1330 bis 1340 entstand vermutlich auf Bestellung König Karl Roberts das sogenannte Ungarische Anjou-Bilderlegendarium, das die Lebensgeschichten Christi und der Heiligen nicht in der Kalenderordnung der Feste, sondern der Hierarchie der Heiligen folgend in Bildern erzählt. Das Werk ist nur fragmentarisch erhalten und zudem auf sechs verschiedene Sammlungen verteilt, wobei jener Teil im Vatikan, in dem sich auch die Gerhardlegende befindet, der umfangreichste ist. Die Hauptquelle der Legenden ist die Legenda aurea, ergänzt um die ungarischen Heiligen. Gerhards Legende ist auf zwei Seiten mit je vier Bildern dargestellt. Die ersten vier Bilder zeigen ihn als Pilger vor König Stephan, als Eremit ein Buch schreibend, seine Bischofsweihe und als Prediger. Die nachfolgenden vier Bilder stellen seinen Tod, die Überführung seines Leichnams nach Tschanad und seine Bestattung dar. Zwei andere Märtyrerbischöfe, Stanislaus aus Polen und Thomas von Canterbury, die im Kodex vor und nach Gerhard dargestellt werden, erhielten ebenso viele Bilder.

\section{Gedenk- und Kultstätten}

Die Erinnerung an den heiligen Bischof Gerhard wird hauptsächlich in seiner Geburtsstadt Venedig und auf dem Schauplatz seines Wirkens, in Ungarn, gepflegt. Sein venezianischer Kult wird hier nicht behandelt, doch es muß erwähnt werden, daß in Ungarn 
Gerhards Reliquien zusammen mit seinen Hauptkultstätten im Laufe der Jahrhunderte zugrunde gegangen waren und alle seine heute bekannten Reliquien in Murano erhalten geblieben sind. Von dort kehrten einige während des 19. und 20. Jahrhunderts nach Ungarn zurück. In Ungarn gedenkt man Gerhards jährlich am 24. September im Rahmen der Liturgie im ganzen Land, an drei Schauplätzen seines Lebens aber ist das Gedächtnis an ihn besonders lebendig: in Bakonybél, wo er als Eremit lebte, in seinem Bistum und am Ort seines Martyriums.

In Bakonybél, wo er beiden Legenden gemäß sieben Jahre als Eremit lebte, entstand eine kleine Gedenkstätte. Die Legenda maior verbindet einen liebenswerten hagiographischen Topos mit seiner Person: Ein Reh und eine verletzte Wölfin fanden bei ihm Schutz. Der heutige Ort ist ein beliebtes Ausflugs- und Urlaubsziel, wo eine Allee zu der 1825 dem heiligen Gerhard geweihten Kapelle führt. Vor der Kapelle wurde anläßlich des 950. Todestages des Heiligen 1996 eine Statue aufgestellt, das Werk Sankt Gerhard und das Reh von István Szabó.

Für den Kult des heiligen Gerhard war bis zum Ende des Mittelalters Tschanad, der Sitz des Bistums und Gerhards Bestattungsort, am wichtigsten. Die Legenda maior beschreibt die Geschichte der Überführung von Gerhards Leichnam von Pest nach Tschanad, zu der es angeblich sieben Jahre nach seinem Martyrium kam. Der Legende gemäß brach zwischen dem Kloster und dem Kapitel ein Streit darüber aus, ob er im Dom oder im Kloster bestattet werden solle. Schließlich beendete ein Wunder die Auseinandersetzung zugunsten des Klosters. Gemäß der Legenda maior sei Königin Elisabeth, die für ihre Religiosität und auch für ihre eifrigen Kloster- und Kirchengründungen bekannt war, 1361 durch das Verdienst des heiligen Gerhard von einer Krankheit genesen. Deshalb ließ sie aus Gold und Silber eine Grabstätte im Kloster errichten, wo die Reliquien des Heiligen aufgehoben werden sollten. Die Jahreszahl 1361 ist, wie alle Jahreszahlen in der Legende, nicht eindeutig belegbar.

Belegt ist dagegen, daß sich Bischof György Kapronczai, der auch am Königshof in diplomatischen Diensten stand, bereits 1345 an Papst Clemens VI. wandte und um Ablaß für die den Dom besuchenden Gläubigen an den Festen des heiligen Georg, Namenspatron des Doms, und des heiligen Gerhard bat. Auch Gerhards Reimoffizium, das seine Tugenden betont, kann für den Dom verfaßt worden sein. 1514 wurden in dem von György Dózsa geführten Bauernkrieg Kloster und Dom angegriffen. Im April 1526 meldete der päpstliche Gesandte Antonio Burgio, in Ungarn wisse niemand, wo sich die Reliquien des heiligen Gerhard befänden. Daraus kann der Schluß gezogen werden, daß die Reliquien zur Zeit des Bauernkrieges verschwanden. Dagegen berichtete 1519 Domherr Clemens, der in Rom die Gerhard-Legende neu bearbeiten ließ, daß sich Gerhards Gebeine im Dom befänden. 1526 verwüsteten die Osmanen Tschanad, damals wird die Spur der dort aufbewahrten Reliquien endgültig verloren gegangen sein. 1552 nahmen die Osmanen die Stadt ein, was nicht nur das Ende von Tschanad als Bischofssitz bedeutete, sondern auch das Ende der mittelalterlichen Stadt: Diese wurde während der osmanischen Herrschaft in einem Maße zerstört, daß heute nicht einmal ihre Topographie rekonstruierbar ist. 
Der Kult Gerhards ging zwar nicht mit seinem Bistum unter. Mit der osmanischen Herrschaft, in deren Folge ein Bevölkerungsaustausch in Südostungarn stattfand, kam es jedoch zu einem tiefen Bruch in der Verehrung des Märtyrerbischofs. Die Erinnerung an den Heiligen wurde vor allem durch die Jesuiten wachgehalten. Die erste bekannte Darstellung des Heiligen in der Frühen Neuzeit stammt aus der ersten katholischen Übersetzung der Bibel ins Ungarische aus der Hand des Jesuiten György Káldy (1626). Gerhard wurde auf dem Titelblatt als einer der drei wichtigsten ungarischen Bischöfe mit den Attributen eines Bischofs, dem Ornat und dem Hirtenstab, und dem Symbol des Märtyrertums, der Lanze, abgebildet. 1668 erschien die Sammlung der Salzburger St. Peter-Abtei mit 365 Heiligenbildern, die in den Andachtsübungen der Mitglieder der marianischen Kongregation, eine Laienorganisation der Jesuiten, verwendet wurden, indem sie unter den Mitgliedern regelmäßig getauscht beziehungsweise auf den Zusammenkünften der Mitglieder verlost wurden. Gerhard erscheint in diesen Darstellungen als Marienverehrer. Die zahlreich erhaltenen Andachtsbilder aus dem 17. und 18. Jahrhundert weisen auf die Verehrung des Heiligen im Rahmen des Regnum Marianum hin. Während der Herrschaft Maria Theresias wurde auch der Versuch unternommen, den heiligen Gerhard als Verfechter der katholischen Kirche hervorzukehren. Das Komitat Tschanad erhielt 1761 von der Königin ein neues Wappen, in dessen Mittelpunkt Gerhard mit den bischöflichen Attributen und mit denen seines Martyriums abgebildet wird. Als infolge des Vertrags von Trianon im Jahr 1920 der größere Teil des Komitats Rumänien zugesprochen wurde, schaffte man zwar das ungarische Wappen ab; aber nach der politischen Wende 1989 nahm das ungarische Komitat Csongrád, zu dem heute auch ein kleiner Teil des historischen Komitats Tschanad gehört, das alte Wappen mit Gerhards Gestalt in seinem neuen Wappen wieder auf.

Die katholische Kirche pflegte den Gerhard-Kult im 19. Jahrhundert weiter, doch erst anläßlich des neunhundertjährigen Jubiläums der Christianisierung Ungarns erhielt die Erinnerung an Gerhard einen neuen Aufschwung. Im Jahr 1900 veranstaltete der Tschanader Bischof Tamás Dessewffy eine große Wallfahrt von Timişoara, dem Sitz des Bistums, zu den Reliquien des heiligen Gerhard nach Murano und in die Kirche auf der venezianischen Sankt-Georg-Insel, wo er eine Gedenktafel zu Ehren des Heiligen anbrachte. 1901 entstand auch der Plan, am Gellértberg, jenem Ort in Budapest, wo Gerhard sein Martyrium erlitten hatte, ein imposantes Denkmal zu errichten. Hier war Gerhard der Legenda maior gemäß von den Heiden in einem Karren in die Tiefe hinabgestoßen worden; bereits im 13. Jahrhundert hatte man begonnen, den Berg - als Kelenberg oder deutsch damals auch als Blocksberg bekannt - nach dem heiligen Gerhard zu benennen. 1904 konnte der Plan realisiert werden: An der zur Donau gerichteten Seite des Berges wurde eine sieben Meter hohe Bronzestatue Gerhards aufgestellt, die rasch zu einem Wahrzeichen Budapests wurde. Das Werk von Gyula Jankovits steht in der Mitte einer Halbkreisarkade und zeigt den Missionsbischof, der in seiner erhobenen rechten Hand ein Kreuz gen Himmel hält. Hier wurde das ikonographische Attribut der Lanze oder auch der Palme mit einem neuen Element, dem Kreuz, erweitert. Die Kosten der Statue, 


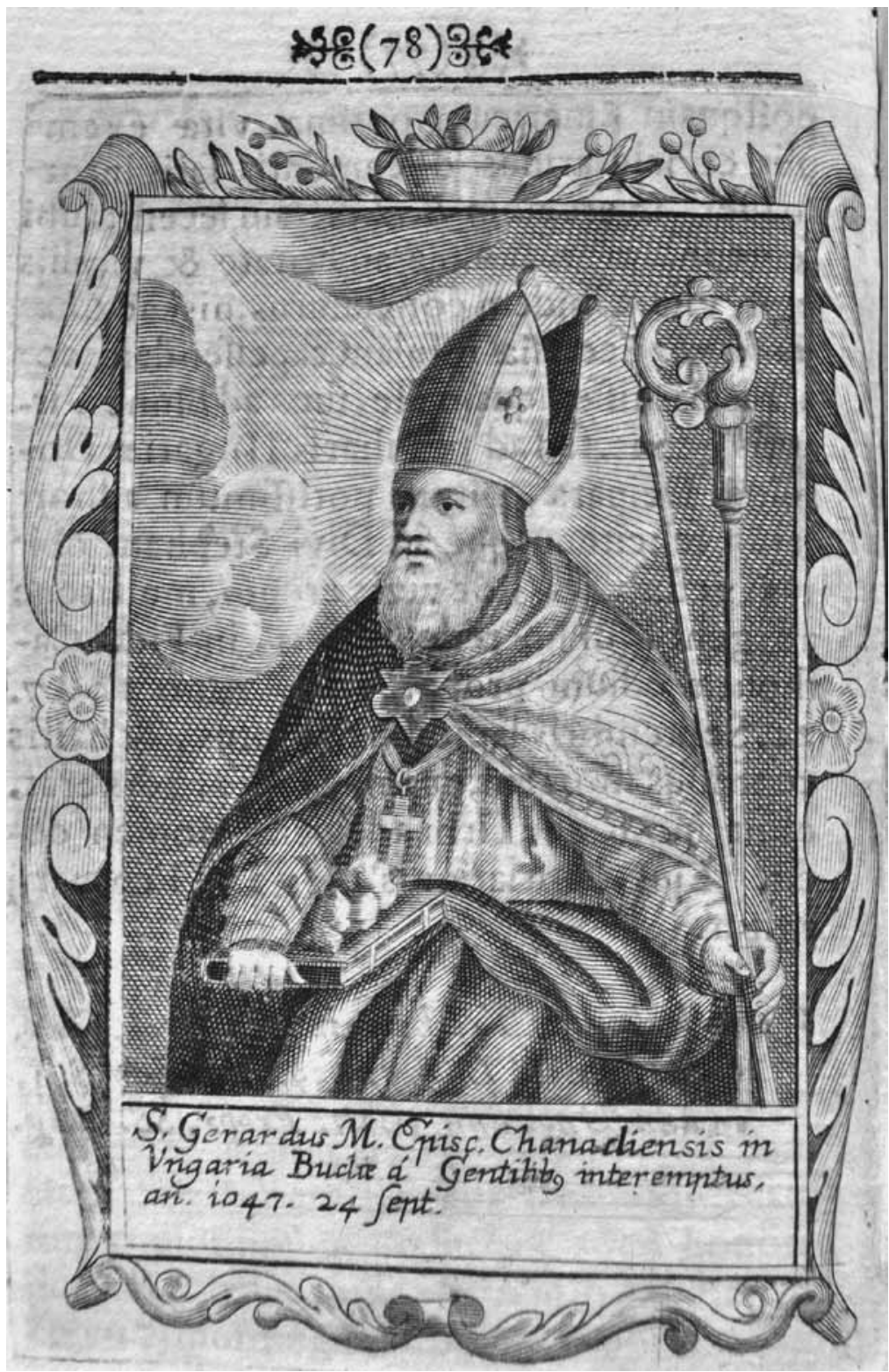

Der Jesuitenpater Gábor Hevenesi (1656-1715) veröffentlichte 1692 ein Werk über die ungarischen Heiligen. Von den Kupferstechern Schott und Hoffmann in Wien ließ er etwa fünfzig Bilder anfertigen, die in der späteren ikonographischen Darstellung der ungarischen Heiligen von großer Wirkung waren. Der heilige Gerhard wird im Bischofsornat, in seiner rechten Hand mit einem Buch als Symbol seiner literarischen Tätigkeit, abgebildet, wobei die auf dem Buch plazierten Steine auf sein Martyrium verweisen. In seiner linken Hand hält Gerhard den Hirtenstab und die Lanze. In Hevenesis Werk erscheint somit zum ersten Mal das ganze Ensemble von Gerhards späteren ikonographischen Merkmalen. Bildnachweis: Hevenesi, Gabriel: Ungaricae Sanctitatis indicia [...]. Tyrnaviae 1692, 78. 
welche die einst enge Verflechtung von Christentum und Monarchie symbolisiert, übernahm Kaiser Franz Joseph I.

Heute tragen nicht nur der Berg, sondern auch ein Platz und ein Bad am Fuße des Gellértbergs den Namen des Bischofs. An jener Stelle, an dem sein Kopf laut der Legenda maior auf einem Stein zertrümmert wurde, baute man eine ihm geweihte Kirche. Die seit dem 13. Jahrhundert in den Quellen häufig belegte Pfarrkirche war allerdings am Ende des Mittelalters schon verschwunden, heute ist nicht einmal ihre Stelle genau identifizierbar. Ein später Nachfolger dieser Kirche ist die 1992 geweihte Pfarrkirche des heiligen Gerhard im heutigen Stadtteil Kelenföld. An ihrem Giebel befindet sich eine Statue mit der Darstellung des Martyriums des heiligen Gerhard von Imre Varga. Zur Zeit des Martyriums im 11. Jahrhundert existierte nur eine Pfarrkirche, die König Stephan I. hatte bauen und zu Ehren der Jungfrau Maria weihen lassen. Sie stand an der Pester Fähre auf dem rechten Donauufer, heute am Pester Brückenkopf der Elisabethbrücke. Am Tage nach Gerhards Ermordung bestatteten ihn der Legenda minor gemäß „fromme Männer“ in der dortigen Kirche. In der Pfarrkirche Assumptio Mariae wird seit neuester Zeit die Verehrung des Heiligen wieder aktiv betrieben. So wurde zum 950. Todestag Gerhards auf Initiative der Pfarrkirche eine Wallfahrt nach Venedig unternommen. Im Jahr 2000 regte der Pfarrer der Kirche die Überführung eines Reliquienteils Gerhards aus Murano nach Budapest an. Die erhaltene Reliquie wurde im Marmorblock des neuen VersusPopulum-Altars, der am 23. März 2002 geweiht wurde, untergebracht. Seither gilt die Kirche als ein nationales Heiligtum.

Nach dem Vertrag von Trianon wurde Szeged 1923 der neue Bischofssitz, wo Gerhard in dem zwischen 1879 und 1930 erbauten Dom einen anspruchsvollen Altar erhielt. Die Statue von Nándor Horánszky zeigt ihn im Bischofsornat mit seinem Schüler, dem heiligen Emerich. In Szeged fand auch 1946 die Feier anläßlich des 900. Todestags Gerhards statt, die zugleich als eine kirchliche Demonstration gegen die drohende Diktatur gedacht war. 1930 wurde auch Timişoara in Rumänien zu einem selbständigen Bistum erhoben, jedoch 1948 von der rumänischen Regierung zum Dekanat degradiert, weshalb die Bischöfe heimlich geweiht werden mußten. Erst 1990 erhielt es seinen Bistumsrang zurück. Im Jahr 2000, als die römisch-katholische Kirche das zweitausendjährige Jubiläum der Christenheit feierte und die ungarische Kirche gleichzeitig ihr Millennium beging, veranstalteten Endre Gyulai, Bischof von Szeged-Tschanad, und Martin Roos, Bischof von Timişoara, im Sinne des gemeinsamen Gerhard-Kultes zusammen eine Wallfahrt zum Grab des heiligen Gerhard, das sich längst nicht mehr in Tschanad, sondern Dank des venezianischen Kultes in der Muranoer Basilika Santi Maria e Donato befindet. 


\title{
V. Auswahlbibliographie
}

\author{
a) Werke
}

BATTHYÁNY, Ignác (Hg.): Sancti Gerardi episcopi Chanadiensis scripta et acta hactenus inedita cum serie episcoporum Chanadiensium. Albo-Carolinae 1790; SILAGI, Gabriel (Hg.): Gerardi Moresanae aecclesiae seu Csanadiensis episcopi Deliberatio supra hymnum trium puerorum. Turnout 1978; KARÁCsONYI, Béla/Szegfü, László (Hg.): Deliberatio Gerardi Moresanae aecclesiae episcopi supra hymnum trium puerorum. Elmélkedés. Gellért, a marosi egyház püspöke a három fiú himnuszáról. Szeged 1999.

\section{b) Gerhard-Legenden, liturgische Texte}

Siculus, Simon Iulius: Divus Gerardus episcopus et martyr. Romae 1519; Zent Gellérd pispeknek innepéröl. In: Volf, György (Hg.): Érdy-kódex. Budapest 1876, 324-328; De s. Gerardo Cenadiensi [Officium rhythmicum: Aurea iam saecula]. In: Dreves, Guido Maria (Hg.): Analecta Hymnica 45a. Liturgische Reimoffizien des Mittelalters. Leipzig 1904, 95-97; Chronici Hungarici compositio saeculi XIV. In: SzENTPÉTERY, Emericus (Hg.): Scriptores rerum Hungaricarum tempore ducum regumque stirpis Arpadianae gestarum, Bd. 1. Budapest 1937, 336-342; MADZSAR, Emericus (Hg.): Legenda sancti Gerhardi episcopi. In: SzENTPÉTERY, Emericus (Hg.): Scriptores rerum Hungaricarum tempore ducum regumque stirpis Arpadianae gestarum, Bd. 2. Budapest 1938 [Reprint 1999], 461-506; BANFI, Florio: Vita sancti Gerardi episcopi Moresenensis et martiris origine Veneti. In: Benedictina 2 (1948) 262-330; LeVÁRDY, Ferenc: Magyar Anjou Legendárium [Ungarisches Anjou-Bilderlegendarium]. Budapest 1973, XXIII; Gerhardslegenden. In: BogYAY, Thomas von/BAK, János/SILAGI, Gabriel (Hg.): Die heiligen Könige. Graz/Wien/Köln 1976, 77-119; Morello, Giovanni/Stamm, Heide/Betz, Gerd (Hg.): „Ungarisches Legendarium“ (Codex Vat. Lat. 8541), Bd. 1-2. Stuttgart/Zürich 1990; Catrin, Giulio: Musica e liturgia a Sanc Marco, Bd. 3. Venezia 1990, 294-298, 160*-172*; KovÁCs, Andrea/FöLdvÁRY, Miklós: Egy ismeretlen Szent Gellért officium (O felicem praesulem) [Ein unbekanntes Sankt-GerhardOffizium (O felicem praesulem)]. In: Magyar Könyvszemle 126 (2010) 1-23.

\section{c) Darstellungen}

KARÁCSONYI, János: Szent Gellért csanádi püspök élete és művei [Leben und Werk des heiligen Gerhard, Bischof von Tschanad]. Budapest 1887; Dedek Crescens, Lajos: Das Leben des heiligen Märtyrers Gerardus, des ersten Bischofs von Csanád. Budapest 1900; KARÁCsONYI, János: Szent Gellért püspök és vértanú élete [Leben des Bischofs und Märtyrers Gerhard]. Budapest 1925; JuHÁsz, Kálmán: Gerhard, der heilige Bischof von Maroschburg. In: Studien und Mitteilungen zur Geschichte des Benediktiner-Ordens und seiner Zweige 48 (1930) 1-35; MaCARTnEY, Carlile Aibner: The Medieval Hungarian Historians. Cambridge 1953, 152-161; IvÁNKA, Endre: Das „Corpus Areopagiticum“ bei Gerhard von Csanád († 1046). In: Traditio 15 (1959) 205-222; PÁszToR, Edith: Problemi di datazione della „Legenda maior S. Gerhardi episcopi“. In: Bullettino dell'Istituto Storico Italiano per il Medio Evo e Archivio Muratoriano 73 (1962) 113-140; BARRÉ, H[enri] C.S.Sp.: L'œuvre Mariale de Saint Gérard de Csanád. In: Marianum 25 (1963) 262-296; JuHÁsz, Kálmán: Das Tschanad-Temesvarer Bistum im Spätmittelalter 1307-1352. Paderborn 1964; PÁszTor, Edith: Gerardo, vescovo di Csanád. In: Bibliotheca Sanctorum, Bd. 4. Roma 1964, 194-186; KoszToLnYIK, Zoltán: Hungarian cultural policy in the life and writings of Gerard of Csanad. Diss. New York 1969; LeCLERCQ, Jean: Saint Gerard et le monachisme. 
In: Studia Monastica 13 (1971) 13-30; CsóKA, J. Lajos: Szent Gellért kisebb és nagyobb legendájának keletkezéstörténete [Entstehungsgeschichte der kleineren und größeren Legende des heiligen Gerhard]. In: HoRvÁTH, János/SzéKelY, György (Hg.): Középkori kútföink kritikus kérdései. Budapest 1974, 137 145; Jelenits, István: Cena Agni - mensa Christi. Contribution aux raports de la terminologie eucharistique du moyen âge et de quelques phrases importantes de la Passio et de la Legenda Sancti Gerhardi. In: Acta Antiqua Academiae Scientiarum Hungariae 23 (1975) 345-353; GYöRFFY, György: István király és müve [König Stephan und sein Werk]. Budapest 1977, 195-197; BorsA, Gedeon: Az 1519. évben nyomtatott Gellért-legenda [Die 1519 gedruckte Gerhard-Legende]. In: Magyar Könyvszemle 96 (1980) 377-384; Mezey, László: Gellért-problémák [Gerhard-Probleme]. In: Vigilia 45 (1980) 590-598; JELENITS, István: Adalékok legendáink dogma- és liturgiatörténeti vizsgálatához [Beiträge zur dogmen- und liturgiegeschichtlichen Untersuchung der ungarischen Legenden]. In: SzÉKELY, György (Hg.): Eszmetörténeti tanulmányok a magyar középkorról. Budapest 1984, 227-234; HeINZER, Felix: Neues zu Gerhard von Csanád: Die Schlußschrift einer Homiliensammlung. In: Südost-Forschungen 41 (1982) 1-7; GERICS, József: Auslegung der Nacherzählung mittelalterlicher Quellen in unserer Zeit (Bischof Sankt Gerard von Tschanad über König Aba). In: Acta Historica Academiae Scientiarum Hungaricae 32 (1986) 335-348; GYöRFFY, György: Az Árpád-kori Magyarország történeti földrajza, Bd. 1, 4. Budapest 1987, 1998; KlanicZAY, Gábor: Il monte di San Gherardo e l'isola di Santa Margherita: gli spazi della santità a Buda nel Medioevo. In: Boesch Gajano, Sofia/Scaraffia, Lucetta (Hg.): Luoghi sacri e spazi della santità. Torino 1990, 267-284; KlanicZAY, Gábor/MadAs, Edit: La Hongrie. In: Philipart, Guy (Hg.): Hagiographies, Bd. 2. Turnhout 1996, 113-114, 138-140; Tóth, Ferenc (Hg.): In Memoriam Szent Gellért. Makó 1996; Silagi, Gabriel: Gerhard. In: Mariacher, Bruno u. a. (Hg.): Lexikon des Mittelalters, Bd. 4, Stuttgart/Weimar 1999, Sp. 1312; MADAS, Edit: Szent Gellért püspök elveszett homíliái és sermói [Die verlorengegangenen Homilien und Sermone von Bischof Gerhard dem heiligen]. In: DIES.: Középkori prédikációirodalmunk történetéből. Debrecen 2002, 13-48; NIERO, Antonio: Sul culto di San Gerardo a Venezia. In: Érszegi, Márk Aurél (Hg.): San Gerardo fra Venezia e Ungheria. Venezia 2002, 14-37; PÜSPÖKI NAGY, Péter: Szent Gellért püspök-vértanú élete és müve. A Velencéből áthozott ereklye bemutátásának emlékére a Budapest Belvárosi Római Katolikus Főplébánián 2002. március 23. [Leben und Werk des Bischofs und Märtyrers Gerhard, des heiligen. Zur Erinnerung an die Ausstellung der aus Venedig überführten Reliquie in der katholischen innerstädtischen Hauptpfarrkirche von Budapest am 23. März 2002]. Budapest 2002. 